Rapport - Société canadienne d'histoire de l'Église catholique RAPPORT

\title{
Histoire du diocèse du Sault-Sainte-Marie
}

\section{Adolphe Proulx}

Volume 27, 1960

URI : https://id.erudit.org/iderudit/1007404ar

DOI : https://doi.org/10.7202/1007404ar

Aller au sommaire du numéro

Éditeur(s)

La Société canadienne d'histoire de l'Église catholique

ISSN

0318-6148 (imprimé)

1927-7075 (numérique)

Découvrir la revue

Citer cet article

Proulx, A. (1960). Histoire du diocèse du Sault-Sainte-Marie. Rapport - Société canadienne d'histoire de l'Église catholique, 27, 71-82.

https://doi.org/10.7202/1007404ar

Tous droits réservés @ C La Société canadienne d'histoire de l'Église catholique, 1961
Ce document est protégé par la loi sur le droit d'auteur. L'utilisation des services d’Érudit (y compris la reproduction) est assujettie à sa politique d'utilisation que vous pouvez consulter en ligne.

https://apropos.erudit.org/fr/usagers/politique-dutilisation/ 


\section{Histoire du diocèse du Sault-Sainte-Marie}

- Le Christianisme n'est pas une grandeur historique: c'est l'histoire qui est une grandeur chrétienne "- Henri de Lubac.

La Société historique du Nouvel-Ontario a déjà consacré plusieurs fascicules à l'histoire du diocèse du Sault-Sainte-Marie. Il serait téméraire de vouloir répéter ici ce qui a été si bellement exprimé, par exemple, dans le document numéro 6 de la Société. Dans la préface de cette publication, le regretté $\mathrm{M}^{\mathrm{gr}}$ Stéphane Côté écrivait justement: "Les plus belles épopées ne sont pas écrites. Ce document sans prétention fournirait le sujet du plus exaltant des poèmes. La conquête obscure et laborieuse d'un vaste territoire à la foi par une poignée de missionnaires qui ont donné leur vie à la tâche, voilà ce qu'il faut esquisser. L'idéal était haut et la réussite est belle, si l'on considère qu'on a seulement voulu dessiner à grands traits l'histoire des missions nord-ontariennes de 1844 à 1904. Il faudrait des volumes pour développer ce plan. »

Le travail que nous présentons est sans prétention. Nous ne ferons qu'effleurer l'histoire de cette portion de l'Eglise qui eût ses heures héroïques, et qui fut servie par de véritables saints. Le Rév. Père Lorenzo Cadieux, s.j. dans un magistral document a tracé l'évolution de cette contrée sauvage jusqu'à la formation de ce qu'on appelle aujourd'hui les diocèses de Fort William et du Sault-Sainte-Marie. Nul mieux que l'émérite fondateur de la Société historique pouvait raconter l'épopée missionnaire des Fils de saint-Ignace. Nous nous contenterons de rappeler ici à grands traits les lignes maîtresses de cette histoire de notre église diocésaine à ses débuts. Nous puiserons notre documentation aux sources qui ont déjà servi, et nous poursuivrons notre travail par un raccourci de l'histoire du diocèse depuis 1904.

\section{L'ÉPOQUe MISSIONNAIRE.}

Le père Cadieux dans le document $n^{\circ} 6$ de la Société historique divise son exposé en deux parties: Missions indiennes et Missions chez les Blancs. * Les premières, écrit-il, formant un vaste réseau encerclant les lacs Supérieur, Nipissing et la baie Georgienne, étaient desservies par les résidences centrales: Wikwémikong, Sault-Sainte-Marie et Fort William; elles reçurent une vive impulsion en 1844, à l'arrivée des Pères Jésuites. Les secondes, les missions blanches, datent de 1883, lors de la construction du grand transcontinental, le Pacifique Canadien. A cette gigantesque entreprise plusieurs milliers de cheminots travaillèrent. Au passage de cette légion d'hommes, armés de pioches et de pelles, surgissaient des postes, transformés aujourd'hui en paroisses florissantes: North Bay, Sturgeon Falls, Verner, Warren, Markstay, Sudbury, Chelmsford, et nombre d'autres jusqu'à Fort William. (P. 6, Doc. $n^{\circ}$ 6.) 
Les Pères Jésuites reprenaient en 1844 un apostolat commencé deux cents ans plus tôt par le père Joseph-Antoine Poncet dans lî̀le Manitouline. Le père Choné, premier des Jésuites demandé par $\mathbf{M}^{\mathrm{gr}}$ Power, archevêque de Toronto, remplace M. l'abbé Jean-Baptiste Proulx à Wikwémikong. Ce dernier passe une année avec le nouvel arrivé pour l'initier à l'Ojibwé. A la suite du père Choné, ce fut une légion de missionnaires, 42 prêtres et 49 frères coadjuteurs qui rayonnèrent à partir de cette mission de Sainte-Croix de Wilwémikong.

Sera-t-il nécescaire d'illuctrer nar des statictiquıe le dévelnnnement prodigieux de cette église missionnaire bâtie pierre par pierre grâce au dévouement et au zèle indomptable de ces valeureux hérauts de l'Evangile? Eglises, écoles, couvents, école industrielle, surgissent comme par enchantement sur le passage des missionnaires, et viennent clamer à tous, la pérennité de cette jeune église en même temps que la foi inébranlable de ses chrétiens ! L'église de Wilwémikong, construite en pierres, reste un monument à la gloire de Dieu, et des Fils de saint-Ignace, monument qui devra constamment nous rappeler l'origine de l'église diocésaine !

Encore plus à l'ouest un autre centre devait recevoir sa vitalité des missionnaires jésuites. C'est au Sault-Sainte-Marie, d'abord du côté américain, ensuite du côté canadien. "Le Sault-Sainte-Marie évoque un glorieux passé. C'est la porte de l'ouest qui donne sur le lac royal parcouru par les explorateurs tels qu'Allouez. Dablon, Nouvel, Albanel, les fondateurs de villes, les coureurs de bois et toutes ces vaillantes caravanes qui ont pointé leurs canots vers l'ouest, bien avant qu'une compagnie ferroviaire reliât par un ruban d'acier l'Atlantique au Pacifique. 》 (P. 11, Doc. $\mathrm{n}^{\circ}$ 6.)

"C'est en 1641, devant une foule de 2,000 Sauteux, que les pères Isaac Jogues et Charles Raymbault plantèrent à quelque distance des rapides du Sault, le signe de la découverte, une croix. Vingt-sept ans plus tard, les pères Jacques Marquette et Claude Allouez inauguraient la résidence appelée: Sainte-Marie-du-Sault d'où Sault-Sainte-Marie. Trois chapelles y furent érigées à cette époque. Un siècle s'écoulera et les pères reprendront avec joie leur ancienne mission, réalisation d'un rêve, reprise de contact avec cette terre riche en souvenirs de famille. " (Ibid. p. 11.)

Du Sault-Sainte-Marie on desservait une vingtaine de postes indiens, entre autres Garden River, qui deviendra un centre missionnaire vers 1860. Auguste Kohler, jésuite et médecin-chirurgien diplômé de la Sorbonne de Paris, fut le véritable fondateur de Garden River ou Rivière-au-Désert. Son territoire s'étendait sur trois cents milles le long de la rivière Sainte-Marie. "Il visitait Batchewana, Gros-Cap, Michipicoton Island, Ile Parisienne, la Baie de Goulais que l'illustre vicaire apostolique du Haut-Michigan, $\mathbf{M}^{\mathrm{gr}}$ Frédéric Baraga, dota d'une chapelle." (Doc. $n^{\circ}$ 6, p. 15.)

Ainsi peut-on dire que déjà en 1837, tout le littoral du lac Nipissing au lac Supérieur était jalonné "d'une constellation de croix " (Ibid.) Les apôtres de la baie Georgienne évangélisaient notre futur diocèse en se relayant de poste en poste en véritables athlètes du Christ. 
A la tête des grands lacs, au Fort William, s'établit un troisième quartier général missionnaire. Ainsi des chapelles s'échelonnèrent à Sand Point, Grand Baye, Nepigon House, et Ombabika. Le père du Ranquet, le géant de ces missions, parcourait jusqu'à mille milles au printemps et à l'automne pour visiter tous ces postes d'un territoire particulièrement difficile.

\section{Apostolat chez les Blancs.}

Cette terre du Nord-Ontario qu'habitaient depuis des siècles plusieurs tribus d'Indiens et que les missionnaires Jésuites avaient en grande partie instruits dans la foi, était destinée à une expansion extraordinaire. Les trois principaux facteurs de développement furent l'industrie forestière surtout dans le district d'Algoma, la découverte d'un immense gisement cupro-nickelifère dans la région de Sudbury, et le facteur capital, la construction du chemin de fer du Pacifique Canadien. Cette voie ferrée que l'on a dû coucher sur une distance de 900 milles sur un terrain extrêmement accidenté, mit en valeur ce nouveau pays riche à profusion.

La voie ferrée fut construite en quatre tronçons principaux: du lac Nipissing à Sudbury; de Sudbury à Chapleau; de Chapleau à Fort William; et enfin l'embranchement du Sault-Sainte-Marie.

Dès $1882, \mathrm{M}^{\mathrm{gr}} \mathrm{Jamot}$, évêque de Peterborough appelle les Jésuites à son aide. De 1882 à 1886, les pères François-Xavier Santerre, Louis Côté, Hormidas Caron, Richard Baxter, Olivier Nault, Jean-Baptiste Nolin, Joseph Specht, furent chapelains des cheminots du Pacifique Canadien. Sir Thomas Shaughnessy résume en une phrase lapidaire l'opinion générale sur l'œuvre de ces braves chapelains: "Les Jésuites et les Oblats, disait-il, ont été d'importants facteurs dans le maintien du bon ordre des 40,000 hommes, employés à construire la voie ferrée. »

\section{NAISSANCE DES PAROISSES.}

Les premiers colonisateurs blancs ne tardèrent pas à s'enraciner dans cette nouvelle terre ouverte par la voie ferrée. Tous ne continuaient pas avec les ouvriers du Pacifique Canadien vers l'Ouest, mais préféraient plutôt s'arrêter pour s'établir sur une terre propre à la culture et à l'exploitation forestière. Ainsi surgirent des patelins destinés à devenir plus tard, soit de prospères paroisses rurales, soit des villes industrielles. $\mathrm{Ce}$ furent North Bay, Sturgeon Falls, Verner, Warren, Markstay, Sudbury, Chelmsford, Cartier, Chapleau, Port Arthur, Fort William; et sur l'embranchement du Sault-Sainte-Marie: Copper Cliff, Espanola, Massey, Blind River, Thessalon.

En mai 1886, le père J. Sinnett, s.j., devient le premier curé de North Bay, mais quatre mois plus tard, il devait laisser la place au clergé diocésain. A Verner, les pères Côté, Hormidas Caron, J.-B. Meloche et Hudon se succédèrent dans l'exercice du saint ministère. En 1904, le voyageur à destination de l'Ouest pouvait admirer, au travers les vitres du wagon de jolis clochers dominant les stations à 
l'ouest de Sudbury: Chelmsford, Chapleau, White River, Schreiber, Nepigon, Port Arthur qui avait alors «la réputation d'une petite Babylone » et Fort William, la ville de l'avenir.

En septembre 1888 on commença à célébrer la messe régulièrement à Sudbury, et à Chapleau tous les quinze jours. De petites églises s'édifièrent à Copper Cliff, Victoria Mines, Nairn, Espanola, Webbwood, Massey, Cutler, Spragge, Blind River et Thessalon. Au Sault-SainteMarie, $\mathrm{M}^{\mathrm{gr}}$ Jamot avait construit une petite cathédrale qu'il avait confiée au Pères Jésuites en 1803.

A partir de 1887 le Sault-Sainte-Marie connut le progrès... canal, travaux du chemin de fer et le pont international.

Les limites du diocèse de Peterborough étaient immenses avec 49, 190 âmes, 59 prêtres, 33 paroisses et 4, hôpitaux. En 1904, Rome amputa le diocèse de Peterborough de tout le Nouvel-Ontario pour former celui du Sault-Sainte-Marie.

\section{Fondation DU DIOCÈse.}

et son premier Évêque, Mrr David J. Scollard (1904-1934).

Le territoire du diocèse du Sault-Sainte-Marie comprenait à ses débuts une partie du vicariat apostolique du Nord-Ontario créé par le Saint-Siège et confié à $M^{\mathrm{gr}}$ Jean-François Jamot, nommé Vicaire apostolique le 24, janvier 1874. $\mathrm{M}^{\mathrm{gr}}$ Jamot était avant sa consécration épiscopale, Vicaire général de l'archidiocèse de Toronto. Il établit ses quartiers généraux d'Evêque missionnaire à Bracebridge où il habita de 1874 à 1882; en cette année, quatre comtés du diocèse de Kingston réunis au vaste territoire du vicariat constituèrent le diocèse de Peterborough. $\mathrm{M}^{\mathrm{gr}}$ Jamot prit possession de son siège épiscopal le 21 septembre 1882 dans la ville de Peterborough.

De 1882 jusqu'à la fin de sa vie en 1886, $\mathrm{M}^{\mathrm{gr}}$ Jamot visita regulièrement les postes missionnaires confiés à sa juridiction. Homme d'une grande sainteté et au zèle ardent, il accepta avec allégresse les misères et les soucis inhérents à sa lourde charge pastorale.

$\mathrm{M}^{\mathrm{g}}$ Joseph Dowling succéda à $\mathrm{M}^{\mathrm{er}}$ Jamot sur le siège de Peterborough. Consacré évêque le $l^{\text {er }}$ mai 1887 , il visita dès l'année suivante les missions de la section nord de son diocèse. En ce temps-là, comme nous l'avons vu plus tôt, les Pères Jésuites œuvraient dans toutes les missions, indiennes et blanches, à la seule exception de North Bay, qui avait été confié à l'automne de 1886 à M. l'abbé Joseph Bloem, prêtre diocésain.

En janvier 1889, $\mathrm{M}^{\mathrm{gr}}$ Dowling fut transféré au siège de Hamilton et $\mathrm{M}^{\mathrm{gr}}$ Richard Alphonsus O'Connor devint évêque de Peterborough, et fut consacré dans sa cathédrale le $1^{\text {er }}$ mai 1889. Durant son règne plusieurs églises, chapelles, écoles et presbytères furent construits.

Un flot ininterrompu d'immigrés, de même que le développement des moyens de communication et la découverte de gisements nickelifères, 
rendirent extrêmement difficile la direction de l'extrémité nord du diocèse de Peterborough. Saint Pie $\mathrm{X}$ dans un document en date du 16 septembre 1904, en vertu de son autorité apostolique détacha toute la partie du diocèse de Peterborough au nord du lac Nipissing et de la rivière des Français, y compris l'île sur la baie Georgienne, et constitua le diocèse du Sault-Sainte-Marie, avec siège épiscopal dans la ville du même nom. Dans un deuxième document du même Pie $\mathbf{X}$ en date du 20 septembre, M. l'abbé D. J. Scollard fut élu et nommé premier évêque du nouveau diocèse. Ce dernier, natif de Ennismore, curé à North Bay depuis neuf ans, devait être consacré dans la cathédrale de Peterborough en la fête de saint Mathias de l'année suivante.

Le 2 mars 1905, il revint à North Bay où il fut reçu en triomphe par ses anciens paroissiens. Il continua sa route cependant jusqu'au Sault-Sainte-Marie, pour y être officiellement reçu dans la cathédrale du Sacré-Coeur, sous la direction des Pères Jésuites. On ne s'étonnera pas de voir le premier évêque de ce vaste diocèse reçu par les Pères Jésuites dans sa propre cathédrale! N'eut-ce été l'humilité traditionnelle des Fils de saint Ignace et leur hésitation à accepter l'épiscopat, le premier évêque du Sault-Sainte-Marie eut sans doute été un Jésuite! $\mathbf{M}^{\mathrm{gr}}$ Scollard n'évinça pas les Jésuites de sa cathédrale, mais au contraire " il crut bon de laisser aux bons Pères le soin de continuer leur apostolat et retourna aussitôt à North Bay pour y continuer à exercer lui-même son ministère dans la paroisse Saint Mary tout en assumant ses nouvelles fonctions épiscopales" (Archives personnelles de $\mathrm{M}^{\mathrm{gr}}$ Scollard). A la fondation du diocèse, il $y$ avait six prêtres séculiers, MM. les abbés C. Langlois et 0 . Dupuis à Sturgeon Falls; J.-A. Lecuyer à Verner; S. E. Nayl à Saint-Charles, S. Côté à Blezard Valley et T. J. Crowley à North Bay. De ces six prêtres présents à la fondation du diocèse, un seul reste vivant et encore actif, $\mathbf{M}^{\mathrm{gr}} \mathrm{T}$. J. Crowley, $\mathrm{P}$. A., curé actuel de la paroisse du Précieux-Sang, cathédrale du diocèse dans la ville du Sault-Sainte-Marie.

$\mathrm{M}^{\mathrm{gr}}$ D. J. Scollard devait continuer l'œuvre des évêques de Peterborough et surtout des missionnaires Jésuites qui avaient bâti cette église diocésaine pierre par pierre. Il travailla dans ce diocèse sans relâche jusqu'à sa mort en 1934. Il présida plusieurs assemblées de fidèles qui désiraient construire des églises paroissiales. Il bénit de nombreuses églises, chapelles, écoles, couvents et hôpitaux dans toute l'étendue de ce diocèse qui partait de North Bay pour aller jusqu'à la tête des grands lacs, au Fort William. Ce pasteur à la robuste santé et au zèle ardent, se dépensa sans mesure pour la sanctification de ses ouailles, prêtres et laïques. Il se fit quémandeur auprès de ses confrères de l'Episcopat afin d'obtenir plus de prêtres pour les nombreuses paroisses en formation. Il ne se déroba jamais à ses devoirs pastoraux. Les anciens se souviennent encore du charme et de la force de son éloquence vibrante et persuasive.

Sera-t-il nécessaire d'énumérer les institutions qui jalonnèrent pour ainsi dire les années d'expansion de ce diocèse? Nous faudra-t-il nommer les prêtres, les religieux et les religieuses qui marquèrent de 
leur zèle et de leur charité rayonnante chaque étape de l'organisation diocésaine?

L'œuvre commencée par les missionnaires devait se continuer sous l'impulsion dynamique de l'évêque. Des prêtres vinrent de toutes les régions du Canada, quelques-uns pour y demeurer, d'autres pour prêter au jeune diocèse quelques années de leur vie sacerdotale. $M^{\mathrm{gr}} \mathrm{D}$. J. Scollard aime à rappeler dans son journal, chaque arrivée dans le diocèse de même que le lieu d'origine du nouveau venu. C'est tantôt

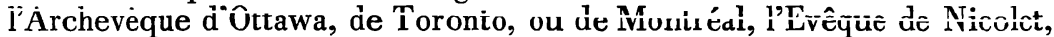
de Joliette ou de Charlottetown qui mérite l'éloge de Monseigneur pour l'aide précieuse qu'ils consentent tour à tour à envoyer au diocèse du Sault-Sainte-Marie.

Les institutions se développent à un rythme régulier. Elles viennent parfaire le travail des missionnaires et épauler les curés dans leur œuvre de sanctification. Les Sœurs Grises de la Croix d'Ottawa, parmi les communautés féminines sont les premières à venir s'établir dans le diocèse pour y ouvrir un hôpital dans ce qu'était alors le village de Sudbury. C'était même avant la fondation du diocèse, en 1896. Les Sœurs Grises devaient également ouvrir un hôpital deux ans plus tard au Sault-Sainte-Marie. Les Pères Jésuites, non contents d'avoir été les ouvriers de la première heure dans l'évangélisation des Indiens et dans l'apostolat chez les premiers colons blancs, ouvrirent un collège à Sudbury pour l'éducation de la jeunesse catholique de langue française dès 1913. Les Filles de la Sagesse ouvraient à Sturgeon Falls leur pensionnat dès l'année de la fondation du diocèse en 1904 et en 1928 construisaient un hôpital. Les Sœurs de Saint-Joseph, d'abord du diocèse de Peterborough, ensuite du diocèse du Sault, fondèrent hôpitaux, collèges pour filles, académies, de North Bay à Fort William, partout où les besoins se firent sentir. Dans le diocèse actuel du Sault-Sainte-Marie, elles ont le mérite de fournir le personnel au plus grand nombre d'hôpitaux catholiques, soit à North Bay, Sudbury, Blind River, et Elliot Lake.

Ce diocèse est cosmopolite dès ses débuts. Les premiers habitants sont Indiens et d'origine française et irlandaise. Mais ce sont bientôt des Slovaques, des Ukrainiens, des Italiens, des Polonais qui viennent grossir le nombre des fidèles catholiques. $\mathbf{M}^{\mathrm{gr}}$ J. D. Scollard ouvre la première église slovaque du diocèse à Fort William en 1907, la première église ruthène à Copper Cliff en 1909, la première église italienne au Sault-Sainte-Marie en 1910. A North Bay même, il est impératif de construire une nouvelle résidence épiscopale. Le 14 février $1915, \mathrm{M}^{\mathrm{gr}}$ Scollard déménage dans la résidence actuelle, sur la rue McIntyre, dans ce que les habitants auront l'habitude d'appeler, "le Palais». Cette résidence devait être suffisamment spacieuse pour loger tous les prêtres séculiers à l'occasion de la retraite annuelle.

Le premier vendredi du mois de septembre 1934, le premier évêque du Sault-Sainte-Marie, mourait subitement dans la Banque Royale de North Bay. Il avait travaillé pendant trente-neuf ans dans cette partie du diocèse qu'il avait vu grandir comme le grain de sénevé de l'Evangile. 


\section{DeUXIÈME ÉVÊQUE DU DIOCÈSE.}

$M^{\text {or }}$ Raphä̈l Hubert Dignan, (deuxième évêque du Sault-SainteMarie) (1934-1959) du diocèse de London fut élu évêque du diocèse du Sault-Sainte-Marie la veille de Noël 1934, et fut consacré évêque le 19 mars de l'année suivante dans la cathédrale de London. Le 2 avril 1935 il arrivait à North Bay. Il semble bien que les évêques du Sault-Sainte-Marie ne devaient pas déménager dans la ville du Sault mais continuer à diriger leur diocèse de la ville de North Bay !

$\mathbf{M}^{\mathrm{gr}}$ Raphaël Hubert Dignan devait continuer l'œuvre de son prédécesseur, ouvrir de nouvelles paroisses, appeler de nouvelles communautés dans le diocèse, affermir l'organisation diocésaine. En 1936, il obtint des Sœurs du Précieux-Sang de London d'ouvrir un monastère à North Bay. C'était les premières sœurs cloîtrées à venir si loin dans le Nord-Ontario perpétuer leur apostolat de la prière et affermir ainsi par la base l'église diocésaine, à l'avenir si prometteur. En 1936, après avoir visité tout le diocèse, $M^{\mathrm{gr}}$ Dignan décide de changer les titulaires de plusieurs églises après avoir obtenu un indult de la Congrégation des Rites. C'est ainsi que la pro-cathédrale prit le titre de l'Assomption et que la cathédrale dans la ville du Sault changea son titre de Sacré-Cour en celui du Précieux-Sang, et que l'église Saint-Joseph à Sudbury devint l'église du Christ-Roi.

$\mathrm{M}^{\mathrm{gr}}$ Dignan voulut également détacher les Sœurs de Saint-Joseph de la communauté de Peterborough. Il réussit en 1936 et ce fut le début de la communauté diocésaine des Sœurs de Saint-Joseph, avec leur maison mère à North Bay.

Le règne de $\mathrm{M}^{\mathrm{gr}}$ Dignan fut marqué par la division de ce diocèse devenu trop grand. Toute la partie du diocèse à l'ouest du $86^{\circ}$ parallèle, jusqu'à la frontière du Manitoba forma le nouveau diocèse de Fort William, le 14 mai 1952 , avec comme premier titulaire $\mathbf{M}^{\mathrm{rr}}$ Edward Jennings.

Les paroisses qui étaient sous la direction des Religieux, surtout ceux de la Compagnie de Jésus passèrent, une à une, au clergé séculier. Les Pères Jésuites conservèrent Sainte-Anne à Sudbury et les missions indiennes dans lesquelles ils avaient travaillé depuis les tout débuts. Ces fondateurs de diocèse ne s'agrippèrent pas aux paroisses de ce coin de pays mais consentirent de bonne grâce à laisser au clergé séculier ces églises, ces résidences, qu'ils avaient édifiées au prix de multiples efforts et de pénibles sacrifices.

Le deuxième évêque du Sault-Sainte-Marie appela dans son diocèse les Rédemptoristes pour diriger une paroisse pour les fidèles de langue anglaise à Minnow Lake en même temps qu'une résidence pour leurs missionnaires; les Basiliens pour une école secondaire à Sudbury et au Sault-Sainte-Marie; il aida grandement à l'œuvre des Résurrectionnistes à North Bay, où ces derniers avaient ouvert un Collège pour garçons à l'invitation de $\mathrm{M}^{\mathrm{gr}}$ Scollard en 1931. Les Sœurs de l'Assomption de Nicolet avaient également été appelées par le premier évêque du diocèse à prendre la direction des écoles bilingues dans la ville de North Bay. 
Homme extrêmement pratique, $M^{e r}$ R. H. Dignan administra le diocèse d'une façon très rationnelle. Chaque paroisse devait être en mesure de subvenir à la subsistance des prêtres appelés à la servir de même que de payer les dettes contractées dans la construction de l'église et du presbytère. Il ne manqua jamais d'insister sur l'importance du ministère strictement pastoral en appuyant sur la vie sacramentelle. Il rappelait toujours avec orgueil que le diocèse du Sault-Sainte-Marie arrivait toujours le premier quant aux nombres de communions distribuées durant l'année.

Le deuxième évêque du Sault-Sainte-Marie devait travailler dans ce vaste diocèse jusqu'en novembre 1957. Durant les dernières années de son administration, il souffrait d'une affection cardiaque. En décembre 1956, il demanda au Saint-Siège un évêque auxiliaire pour le seconder dans sa lourde tâche. Le 12 décembre de la même année, les journaux annonçaient l'élection de Son Excellence $\mathbf{M}^{\mathrm{gr}}$ Alexander Carter comme évêque coadjuteur du diocèse avec droit de succession. Dès le 7 mars de l'année suivante, $\mathrm{M}^{\mathrm{gr}}$ Carter, consacré évêque par Son Eminence le Cardinal Paul-Emile Léger dans l'église Notre-Dame de Montréal le 2 février, était solennellement reçu dans la pro-cathédrale de l'Assomption à North Bay.

\section{Le troisième Évêque du diocèse: M $^{\text {gr }}$ AleXander Carter.}

Ainsi arrivons-nous en pleine actualité! Quand $\mathrm{M}^{\mathrm{gr}} \mathrm{A}$. Carter arriva dans ce diocèse du Nord-Ontario, que vit-il ? Il vit un diocèse en pleine expansion avec tous les problèmes propres à son organisation à tous les niveaux. Plus de 150,000 fidèles, 215 prêtres dont 122 séculiers, 8 hôpitaux, 4 écoles secondaires pour garçons, 5 couvents pour jeunes filles, l'Université, une dizaine d'écoles secondaires mixtes, 5 écoles de gardes-malades, une école residentielle pour les Indiens, 81 paroisses et 14 missions, voilà en statistiques, la photographie du diocèse.

Dès son arrivée dans le diocèse, $M^{g r} A$. Carter fit une étude sérieuse des problèmes qu'il dut affronter moins d'une année après son arrivée. En effet, le 22 novembre 1957, le Saint-Siège le nomma Administrateur apostolique, " sede plena " et il assuma totalement la direction du diocèse.

S'il est difficile pour un jeune abbé de traiter de l'histoire de son diocèse et de passer quelques jugements sur les événements passés, il semble téméraire et imprudent de traiter du travail ou de l'œuvre de celui qui se trouve son supérieur immédiat. Comme je connais la patience et la bonté de Son Excellence, je me risque tout de même à esquisser un bref jugement puisque le bon historien doit non seulement relater l'histoire mais donner aussi son interprétation des faits! Disons que je me risque à prévoir ce que pourra dire l'histoire dans vingt-cinq ans, quand, de nouveau, la Société Canadienne de l'Histoire de l'Eglise Catholique tiendra ses assises à Sudbury !

Nul autre que $\mathrm{M}^{\mathrm{gr}}$ Carter devait être mieux préparé pour la tâche qui l'attendait. Ayant vécu dans la Métropole du Canada où il exerça des fonctions variées, connaissant à fond l'histoire de son pays, et imbu 
d'un esprit de justice à l'endroit de tous, le Pasteur de notre diocèse a pris en mains dès son accession au siège épiscopal tous les problèmes qui y sont particuliers.

\section{Problème de RaCes.}

Comme vous l'avez constaté, le Nouvel-Ontario s'était peuplé de différentes races dès le commencement de la colonisation.

Dès les débuts, les Canadiens français formaient la majorité des fidèles. Venus du Québec, ils apportaient ici leur foi robuste et leur désir de conserver en même temps que l'héritage religieux, l'héritage de leur culture et de leur langue. Dans un contexte d'une province en immense majorité anglaise et protestante, cette détermination devait leur attirer beaucoup d'ennuis. Le Saint-Siège, à cause sans doute de l'incertitude de la composition démographique de ce territoire dans l'avenir, et à cause de ce contexte ontarien, a cru sage de nommer ici des chefs spirituels de langue anglaise. Comme l'histoire est naturellement indiscrète, je ne crois pas qu'il soit malséant de rappeler ici que les fidèles de langue française, tout en conservant dans l'ensemble leur fidélité à l'évêque que la Providence leur désignait, ont souvent manifesté leurs regrets de ne pas être toujours compris de leur Pasteur. Cette incompréhension fut-elle réelle ou imaginaire? Il n'est pas injurieux de rappeler que les deux premiers évêques du Sault-Sainte-Marie ont peut-être parfois paru injustes, tout en travaillant sincèrement pour la plus grande gloire de Dieu. Ils perdaient patience devant certaines attitudes et réclamations des catholiques de langue française. Il faut ajouter, à la décharge de ces deux premiers évêques, que certains clercs et certains laïques ont parfois été fort maladroits dans la présentation de leur griefs, et que l'intention de certains n'était pas toujours des plus honnêtes. Cependant, la volonté des fidèles de langue française de rester fidèles à leur héritage culturel n'a pas toujours été comprise de leur chef spirituel et fut la cause de malaises détestables pour la paix de l'église diocésaine.

La divine Providence devait préparer un homme qui, parfaitement au courant de notre histoire, et connaissant non seulement la langue mais l'esprit de notre groupe ethnique devait réussir en peu de temps à redresser certains torts, même involontaires, et donner justice à tous. Dans ses lettres pastorales, le troisième évêque du Sault-Sainte-Marie, dès le début de son règne, affirme son intention de gouverner le diocèse en tenant compte des droits de chaque groupe ethnique et en proclamant son mandat sur tous ses fils, sans exception. Cé langage, imbu de franchise et de charité chrétienne, était de nature à plaire à tous les fidèles.

Un extrait de la lettre pastorale du 25 janvier 1958 suffira pour illustrer l'attitude de $\mathbf{M}^{\mathrm{gr}} \mathrm{A}$. Carter sur ce problème particulièrement délicat: "Notre diocèse, écrivait-il alors, se situe dans un contexte historique et géographique déterminé. Pour éviter toute équivoque, qu'on Nous permette d'énoncer clairement, une fois pour toutes, notre façon de voir et d'agir. Le diocèse du Sault-Sainte-Marie est un diocèse 
bilingue. Deux grandes cultures sont à la portée de notre peuple, s'il est assez sage pour en profiter. Quoi qu'il advienne, Nous déclarons publiquement que Nous considérons tous les fidèles comme nos propres enfants ayant droit à la même affection et à la même attention. Les nouveaux Canadiens choisiront normalement l'une ou l'autre des deux langues ou même les deux langues officielles du pays; Nous les encourageons à conserver également leur langue maternelle et leur culture propre. Des écoles bilingues et des paroisses françaises existent dans notre diocèse, non par tolérance ou privilège mais par droit. Nous protegerons ce d̉roit avec ìa conviction de servir la justice et d'accomplir notre devoir. Nous proclamons ces choses clairement pour la première et dernière fois, conscient de remplir notre rôle d'évêque catholique et d'agir en toute conformité avec l'esprit de Notre Mère, la Sainte Eglise. " Cette prise de position courageuse et symptomatique des sentiments de Son Excellence devait grandement pacifier les esprits.

\section{LE PROBLÈME SOCIAL.}

Le diocèse du Sault-Sainte-Marie présente également quelques problèmes d'ordre social. Comme on l'indigue clairement dans l'enquête magistrale qui fut menée dans toute l'étendue du diocèse l'an dernier, la majorité des nôtres sont employés à l'extraction du minerai et aux tâches connexes. Il va sans dire que le syndicalisme ouvrier fut un sujet d'inquiétude dès les débuts. A l'automne de 1958, le plus important syndicat du diocèse, celui des Mineurs et Bocardeurs de Sudbury décida de se mettre en grève. Ce syndicat avait été évincé du Congrès du Travail canadien à la suite d'accusation d'allégeance communiste. La majorité des membres de ce syndicat est catholique. Dans sa Lettre pastorale sur la grève et sur la nécessité de venir en aide aux familles des ouvriers, $\mathrm{M}^{\mathrm{gr}}$ Carter proclama clairement la position de l'Eglise et n'hésita pas à faire le partage des responsabilités.

Après avoir rappelé d'une manière générale la position de l'Eglise en matière d'organisation sociale, Son Excellence entre dans le vif du sujet: "Ce qui Nous préoccupe présentement, c'est la situation à Sudbury, un centre important de notre diocèse, où une grève prolongée s'annonce déjà comme une vraie calamité. Le triste et pitoyable exode d'un grand nombre d'ouvriers et de familles est déjà commencé. Une terrible responsabilité retombe sur les épaules des officiers de la Compagnie et du Syndicat: celle de faire tout ce qui est humainement possible pour amener la fin de la grève dans le plus bref délai. Assurément, dans notre siècle émancipé, la Compagnie ne peut plus considérer la main-d'œuvre comme une denrée équivalente au minerai extrait des entrailles de la terre. Les patrons se rendent certainement compte que leurs vieux et fidèles employés verront leurs petites épargnes se dissiper, tandis que les plus jeunes, avec des soucis de l'organisation de leur foyer et peu d'argent en banque se verront acculés à la misère. Il serait intolérable de penser que l'appât du gain soit le seul principe directeur de leur action, et qu'ils permettront une grève prolongée par intérêt mesquin pour eux-mêmes et pour les actionnaires. On se moque de la justice si les officiers de la Compagnie et du Syndicat durcissent leur 
position réciproque pour l'amour d'une victoire vide de sens et au prix de souffrances imméritées infligées aux familles des employés."

« Il est évidemment du ressort immédiat du Syndicat de veiller au bien-être des grévistes. Il est inconcevable de croire que les responsables du Syndicat aient voulu cette grève sans envisager attentivement toutes ses répercussions sur la vie de leurs membres et sans prendre toutes les mesures nécessaires pour en alléger le côté tragique. Nous avons le droit de supposer qu'une bonne somme des quelques millions de dollars payés en cotisations au cours des années passées est maintenant disponible pour secourir les grévistes. Nous présumons que les membres du Syndicat ont reçu un rapport financier annuel sur l'administration de l'argent perçu. Cette pratique est d'élémentaire prudence et un principe fondamental de gouvernement dans une société démocratique. »

M. l'abbé G. Dion de l'Université Laval donnait la lettre de Son Excellence en modèle d'intervention épiscopale dans un problème particulièrement difficile. Non content de mettre en lumière certains principes de doctrine sociale chrétienne, Monseigneur épaula de toute son autorité les Pères Jésuites qui commençaient à ce moment des cours d'extension spécialement destinés aux ouvriers de Sudbury. Il lança également son CEuvre de Charités diocésaines, destinée à soulager les misères humaines et à montrer toute la sympathie de l'Eglise pour les plus éprouvés de ses enfants.

\section{L'ÉDUCATION.}

Dans ce domaine, $\mathrm{M}^{\mathrm{gr}} \mathrm{A}$. Carter en lançant la grande souscription diocésaine dès le mois de mars 1958, a permis aux communautés religieuses qui se dévouent dans le diocèse depuis le début, de fournir à nos jeunes plus de facilité à fréquenter des institutions catholiques.

Il assista le printemps dernier à l'ouverture du Collège Notre-Dame, institution pour les jeunes filles de langue française, un projet mené à bien grâce à $\mathrm{M}^{\mathrm{gr}} \mathrm{A}$. Carter. Les Sœurs Grises voyaient enfin leur rêve se réaliser grâce à l'appui moral et financier du premier Pasteur diocésain. Il a fortement secondé les efforts de la Compagnie de Jésus désireuse de fonder une Université et les Pères Jésuites reconnurent cet appui illimité mardi dernier, le 27 septembre, quand $\mathrm{M}^{\mathrm{gr}}$ Alexander Carter fut officiellement proclamé le Chancelier de l'Université de Sudbury.

Enfin depuis l'arrivée de $\mathrm{M}^{\mathrm{gr}} \mathrm{A}$. Carter, cinq paroisses ont vu le jour, quelque dix églises furent construites, des presbytères et des couvents rénovés ou construits, et une souscription diocésaine lancée avec succès doit rapporter deux millions et demi de dollars. Les Pères Montfortains ont pris la direction d'une importante paroisse canadiennefrançaise, celle de Saint-Vincent-de-Paul à North Bay; les Petites Sœurs de l'Assomption ont ouvert un couvent à Sudbury pour s'occuper des pauvres et des malades; les Stigmatine Fathers ont fondé un couvent au Sault-Sainte-Marie et seront appelés à fonder des Centres d'Information catholique dans cette partie du diocèse; l'Action catholique forme ses cadres et promet des résultats palpables dans un avenir rapproché; 
dans le domaine social, la situation est en partie redressée à Sudbury ou de nouveaux officiers tentent de reprendre le contrôle des mains de sympathisants communistes.

Le Nouvel-Ontario présage un avenir brillant. Dans tous les domaines l'Eglise impose sa présence et propose son enseignement. Certes, il nous manque des prêtres, et l'organisation diocésaine n'est qu'embryonnaire. La divine Providence veille sur nous et ne permettra pas que l'œuvre des missionnaires et des centaines de prêtres, de religieux, de religieuses et de laïques soit freinée. Au contraire, nous prévoyons construire une église diocésaine à la mesure de ce pays. Le nombre des fidèles augmente sans cesse, la qualité de leur foi et de leur génorosité justifie tous les espoirs. Sous la houlette de leur Pasteur bien-aimé, tous, prêtres et laïques, fournissent chacun dans leur sphère leur plus entière coopération. Même, et surtout dans l'apostolat chez les non catholiques, nous prévoyons dans les années qui viennent, des progrès marqués.

En terminant ce travail, nous devons proclamer notre gratitude envers la divine Providence qui a su, grâce à des âmes d'élite, édifier l'église diocésaine du Sault-Sainte-Marie.

Adolphe Proulx, ptre

Chancelier du Diocèse de Sault-Sainte-Marie. 